Ann. Biol. anim. Bioch. Biophys., I974, 14 (4-B), 74I-745.

\title{
VIABILITÉ DES SPERMATOZOÏDES DE BOUC CONSERVÉS ET CONGELÉS AVEC OU SANS LEUR PLASMA SÉMINAL : EFFET DU GLUCOSE
}

\author{
J.-M. CORTEEL \\ avec la collaboration technique de G. BARIL \\ Station de Physiologie de la Reproduction, \\ Centre de Recherches de Tours, I. N.R. A., \\ 37380 Nouzilly
}

\section{RÉSUMÉ}

A partir d'un pool de deux éjaculats, quatre fractiọns de sperme sont prélevées. Deux sont diluées telles quelles dans un milieu à base de lait écrémé contenant ou ne contenant pas de glucose. Avant d'être diluées dans ce même milieu, les deux autres fractions sont lavées et centrifugées dans une solution physiologique additionnée ou non de glucose. Après dilution, les quatre fractions sont refroidies, glycérolées, équilibrées, congelées, dégelées puis incubées pendant deux heures. Le pourcentage de spermatozoïdes mobiles et leur motilité sont estimés après chaque manipulation. Il ressort que le lavage améliore très significativement le pourcentage de spermatozoïdes mobiles et leur motilité avant et après congélation et que l'addition de glucose aux milieux de conservation améliore la motilité après congélation. Une tendance se dégage en faveur d'une interaction entre le lavage et l'addition de glucose, cette interaction n'est toutefois pas statistiquement significative.

\section{INTRODUCTION}

Malgré quelques résultats encourageants (WAIDE et NrWA, I96I; BoNFERT I965 ; NAGASE, I966 ; HAHN, I969 ; CORTEEI et al., I972), la réussite de la congélation du sperme de bouc présente encore quelques difficultés. Pendant deux saisons sexuelles successives (de juillet à mars inclus), nous avons constaté que le taux de spermatozoïdes détruits par la congélation de la semence était proportionnel au volume initial de l'éjaculat $(r=0,9)$ et inversement proportionnel $(r=-0,9)$ à sa concentration en spermatozoïdes (CORTEEI, et BARIL, I974). Ceci suggère une influence néfaste du plasma séminal dans le milieu de conservation sur l'aptitude des spermatozoïdes 
à supporter la congélation. Pour vérifier cette possibilité nous avons congelé le sperme avec ou sans plasma séminal et mesuré l'effet de l'addition de glucose aux milieux de conservation car le glucose est, parmi les hexoses, celui qui est préférentiellement utilisé par le spermatozoïde de bouc (Fukuhara et Nishikawa, I973).

\section{MATÉRIEL ET MÉTHODES}

A partir d'un mélange de 2 éjaculats successifs de boucs de races Saânen ou Alpine, 4 fractions de $0,2 \mathrm{ml}$ sont prélevées. Deux fractions sont diluées dans une solution de Krebs-Ringer-Phosphate contenant ou non du glucose, puis centrifugées ( $\mathrm{n}^{\circ} 3 \mathrm{et} 4$ ). Après centrifugation, les spermatozoïdes sont resuspendus dans un milieu à base de lait de vache écrémé additionné ou non de glucose. Les deux autres fractions de sperme ( 1 et 2) sont diluées telles quelles dans le même milieu (tabl. I). La dilution est telle que chaque $\mathrm{ml}$ contient $\mathrm{r} \times \mathrm{ro}^{9}$ spermatozoïdes. Les manipulations sont effectuées à la température ambiante. Après dilution, les fractions sont refroidies à $+4^{\circ} \mathrm{C}$ puis la concentration de la semence en spermatozoïdes est réduite de moitié par l'adjonction du volume approprié du même dilueur contenant en plus I4 p. Ioo de glycérol (glycérolisation). Après trois heures d'équilibration, la semence est congelée dans les vapeurs d'azote. Dégelée 24 à 48 heures plus tard à $37^{\circ} \mathrm{C}$, elle est incubée à cette température pendant r 20 minutes.

TABLEAU I

Schéma expérimental

\begin{tabular}{c|c|c}
\hline \multicolumn{2}{|c|}{ Traitements } & \\
\hline Lavage & Glucose & Echantillons \\
\hline 0 & 0 & 1 \\
0 & + & 2 \\
+ & 0 & 3 \\
+ & + & 4 \\
\hline
\end{tabular}

Le pourcentage de spermatozoïdes mobiles et leur motilité (échelle de o à 5) sont estimés : après refroidissement $(A)$, après glycérolisation $(B)$, après équilibration $(C)$, au dégel (D) et après I20 minutes d'incubation à $+37^{\circ} \mathrm{C}$ (E). La diminution du nombre des spermatozoïdes mobiles survenue au cours de l'incubation est calculée en pourcent des spermatozoïdes estimés mobiles au dégel : $\mathrm{D}-\mathrm{E} / \mathrm{D} \times$ roo. Les données estimées pour les différents traitements sont comparées après analyse des variances.

\section{RÉSURTATS}

$$
\text { I. - Effet du lavage }
$$

Les pourcentages de spermatozoïdes mobiles des échantillons lavés sont très significativement supérieurs $(P<O, O I)$ à ceux des échantillons non lavés en $A, B$, $\mathrm{C}$ et $\mathrm{E}$. La motilité individuelle des spermatozoïdes est, dans les fractions lavées, très significativement supérieure $(\mathrm{P}<0, \mathrm{O})$ à celle des spermatozoïdes des fractions non lavées en $\mathrm{B}, \mathrm{C}, \mathrm{D}$ et $\mathrm{E}$ (tabl. 2 ). 
TABLEAU 2

Effet du lavage sur le ponvcentage de spermatozoides mobiles et leur motilité avant et après congélation

\begin{tabular}{|c|c|c|c|c|c|c|}
\hline \multirow{2}{*}{$\begin{array}{l}\text { Moments de } \\
\text { l'estimation }\end{array}$} & \multicolumn{3}{|c|}{$\begin{array}{c}\text { Pourcentage de } \\
\text { spermatozoïdes mobiles }\end{array}$} & \multicolumn{3}{|c|}{ Motilité individuelle des spz } \\
\hline & $\begin{array}{c}1+2: \\
\text { non lavés }\end{array}$ & & $\begin{array}{l}3+4: \\
\text { lavés }\end{array}$ & $\begin{array}{c}1+2: \\
\text { non lavés }\end{array}$ & & $\begin{array}{c}3+4: \\
\text { lavés }\end{array}$ \\
\hline A & 68,2 & $* *$ & 77,9 & 3,9 & NS & 4,1 \\
\hline B & 62,1 & ** & 73,0 & 3,4 & $* *$ & 3,7 \\
\hline C & 58,4 & $* *$ & 71,9 & 3,2 & $* *$ & 3,8 \\
\hline $\mathrm{D}$ & 23,2 & NS & 29,8 & 2,6 & ** & 3,3 \\
\hline $\mathrm{E}$ & 12,0 & $* *$ & 26,8 & 1,4 & ** & 2,4 \\
\hline
\end{tabular}

NS : Différence non significative ; $* *: \mathrm{P}<0,01 ; n=20$.

\section{2. - Effet du glucose}

L'addition de glucose aux milieux de conservation est sans effet sur les pourcentages de spermatozoïdes mobiles avant congélation. Par contre, elle améliore significativement la motilité après dégel et cet avantage demeure après incubation (tab1. 3).

\section{TABLEAU 3}

Effet du glucose sur le pourcentage de spermatozoides mobiles et leur motilité avant et après congélation

\begin{tabular}{|c|c|c|c|c|c|c|}
\hline \multirow{2}{*}{$\begin{array}{l}\text { Moments de } \\
\text { l'estimation }\end{array}$} & \multicolumn{3}{|c|}{$\begin{array}{c}\text { Pourcentage de } \\
\text { spermatozoides mobiles }\end{array}$} & \multicolumn{3}{|c|}{ Motilité individuelle des $\mathrm{spz}$} \\
\hline & $\begin{array}{c}1+3: \\
\text { O Glucose }\end{array}$ & & $\begin{array}{l}2+4: \\
\text { Glucose }\end{array}$ & $\begin{array}{c}1+3: \\
\text { O Glucose }\end{array}$ & & $\begin{array}{l}2+4: \\
\text { Glucose }\end{array}$ \\
\hline A & 74,6 & NS & 71,5 & 4,0 & NS & 4,0 \\
\hline $\mathrm{B}$ & 67,2 & NS & 67,9 & 3,7 & NS & 3,5 \\
\hline C & 66,6 & NS & 63,6 & 3,4 & NS & 3,6 \\
\hline $\mathrm{D}$ & 26,2 & NS & 26,8 & 2,8 & $*$ & 3,1 \\
\hline $\mathrm{E}$ & 22,0 & NS & 21,8 & 1,7 & $*$ & 2,0 \\
\hline
\end{tabular}

NS : Différence non significative; $*: \mathrm{P}<0,05 ; n=20$.

\section{3. - Effet cumulé du lavage et du glucose}

Il n'y a pas d'interaction statistiquement significative entre le lavage et l'addition de glucose aux milieux de conservation bien qu'une tendance se dessine dans ce sens. Il en est de même pour le taux de mortalité des spermatozoïdes pendant l'incu- 
bation puisque celui-ci de 47,4 p. Ioo en l'absence de lavage et de glucose est réduit à 6,8 p. roo seulement dans le cas contraire. La viabilité des spermatoïdes (pourcentage de spermatozoïdes mobiles et motilité) est indiqué dans les deux cas par la figure $\mathrm{I}$.

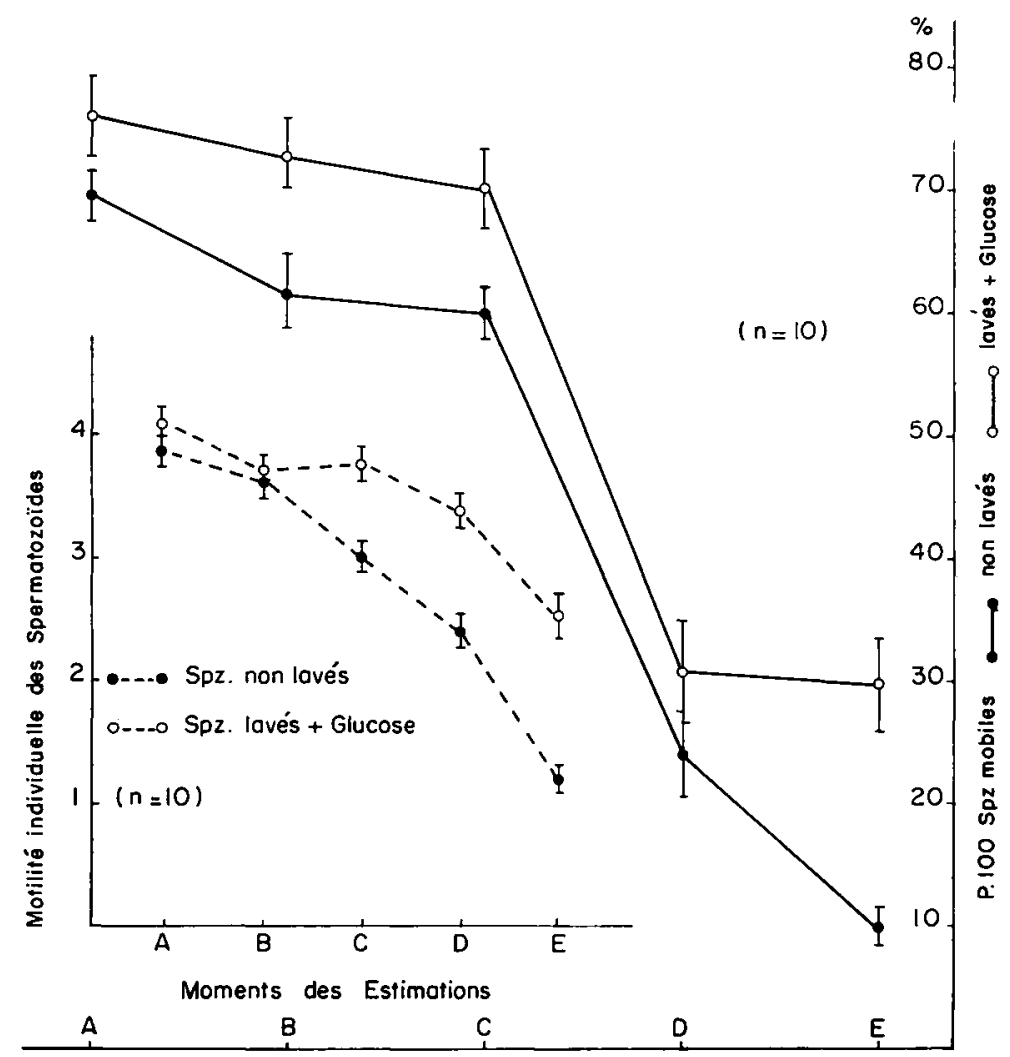

Fig. I. - Effet du lavage et de la dilution en milieux glucosés sur la viabilité des spermatozoïdes de bouc : après refroidissement (A), après glycérolisation (B), après équilibration (C), après décongelation (D) et $\mathrm{I}_{20} \mathrm{O}^{\prime}$ d'incubation (E).

$$
\stackrel{\mathrm{T}}{\mathrm{I}}=m \pm s m
$$

\section{DISCUSSION E'T CONCLUSION}

Dans nos conditions expérimentales, la viabilité des spermatozoïdes débarrassés de leur plasma séminal est avant et surtout après congélation très significativement supérieure à celle des spermatozoïdes demeurés dans leur plasma séminal. Ces résultats sont à rapprocher de ceux de Fukuhara et Nishikawa (I973) pour qui le métabolisme des hexoses, des disaccharides et de la plupart des intermédiaires du cycle de la glycolyse est fortement réduit chez les spermatozoìdes de bouc demeurant dans leur plasma séminal. La meilleure viabilité des spermatozoïdes lavés résulterait 
alors du métabolisme accru du lactose contenu dans le lait et du glucose ajouté aux milieux de conservation. L'exacerbation du métabolisme des sucres après lavage serait une particularité du sperme de bouc car, à notre connaissance, elle n'a pas été signalée dans les autres espèces et notamment chez le bélier (WHITE, I953).

Notre schéma expérimental ne permet pas d'infirmer ou de confirmer l'utilisation préférentielle du glucose par le spermatozoïde de bouc comme l'indiquent FukUhara et Nishikawa (I973). Cependant, la meilleure survie des spermatozoïdes a été obtenue lorsque ceux-ci ont été lavés puis dilués dans des milieux contenant du glucose.

Reçu pour publication en juin 1974.

\section{SUMMARY}

VIABILITY OF GOAT SPERMATOZOA DEEP FROZEN

WITH OR WITHOUT SEMINAL PLASMA : GI,UCOSE EFFECT

Four samples of semen provided by a pool of two successive ejaculates are treated. Two are immediately diluted in a skim milk diluent with or without glucose. Before being diluted the two other fractions are twice-washed in a physiological solution with or without glucose. After dilution, the four samples are cooled, receive glycerol, are equilibrated, deep frozen, thawed and incubated for two hours. The percentage of motile sperm and their motility are evaluated after each of the preceeding steps. Results show that washing of spermatozoa increases the percentage of motile sperm and their motility before and after deep freezing. The addition of glucose to the preservation media increases motility after thawing. The interaction between washing and glucose addition was not statistically significant.

\section{RÉFÉRENCES BIBLIOGRAPHIQUES}

Bonfert A., I965. Possibilités et limites de l'insémination artificielle caprine. Tierzuchter, 17 , I54-I56. Corteel J. M., Courot M., Ortavant R., 1972. Fertility of multiparous goats inseminated with liquid or deep frozen semen after hormonal synchronisation of ostrus before the onset and in the course of the breeding season. VIIth Intern. Cong. Anim. Reprod. Artif. Insem. Munich.

Corteel J. M., Baril G., I974. Résultats non publiés.

Fukuhara R., Nishikawa Y., I973. Effects on pH, sperm concentration, washing and substrate concentration on respiration and motility of goat spermatozoa. Jap. J. Zootech. Sci., 44 (5), 266-27o.

HAHN R., I969. A contribution of the deep freezing preservation of goat buck and ram semen. EAAP meetings, Helsinki, June 23-26.

NAGASE H., I966. In : Neue verfahren under künstlichen besamung der wirbeltiere. VIe Cong. Intern. Reprod. anim. Insem. artif. Paris, I968, 2, 95 I-965.

WAYDE Y., Niwa T., I96r. Storage and insemination trials with frozen semen of goats. Zuchtung breeding Élevage. VIII Cong. Intern. Zootech., Hambourg.

White I. C., r953. The effect of washing on the motility and metabolism of ram, bull and rabbit spermatoza. J. Exp. Biol., 30, 200-2 I3. 